\title{
Preface - Advances in CKD 2020
}

The Renal Research Institute's 22nd International Conference on Dialysis, Advances in Chronic Kidney Disease (CKD) in Los Angeles, CA, Loews Hollywood Hotel, is proud to continue offering an exceptional scientific program, delivered by highly skilled physicians, scientists, and professionals in the field. In recent years, our conference program has grown to include side-by-side to our traditional main conference, a pediatric nephrology symposium and - more recently - a nurse care team track. We made the deliberate decision to integrate a nurse care team track in our conference's program to foster the collaboration between nephrologists, nurses, and researchers.

This year's conference covers a wide range of topics from clinical nephrology to basic science, to the most recent technological developments and novel treatments in acute kidney injury (AKI), CKD, hemodialysis, peritoneal dialysis, kidney transplantation, and epidemiology.

In this issue of Blood Purification, Guven et al. provide an up-to-date review of microcirculation physiology and pathophysiology. The authors describe advanced technological means to analyze the microcirculation at the bedside using hand-held microscopes to visualize capillaries and assess their function. These new tools are suited for clinical application in real time and support decisionmaking in critical conditions such as AKI, sepsis, hypovolemia, and shock. The consequent reduced tissue oxygen availability and hypoxia, which have been observed also in end-stage kidney disease patients on hemodialysis, makes microcirculation a target for therapeutic interventions. The authors posit that the direct visualization of the microcirculation and the use of point-of-care functional analysis will help to identify patients at risk and provide microcirculatory markers for effective therapeutic intervention.
The paper by Fang et al. on intradialytic versus out-ofclinic exercise training addresses the concerns that prescribed low-to-moderate intensity exercise interventions may not be attractive to patients, result in low adherence to exercise programs, and hinder the proven benefits that exercise provides. The authors agree that patients capable of exercising experience improved quality of life (QoL), increased physical functioning, and reduced muscle wasting. Depending on its intensity, intradialytic exercise may improve solute and phosphate clearance. The authors analyze the various types of physical activities and exercise interventions applicable to patients at the dialysis clinic or at home. They strongly recommend personalized interventions and guidance with the patient's active participation in designing and selecting physical activities to improve efficacy and adherence.

Arzhan et al. describe new approaches to the management of restless leg syndrome (RLS) and uremic pruritus, 2 frequently encountered yet poorly understood and elusive to treatment in hemodialysis patients. The authors examine novel treatment options, including pharmacological, nonpharmacological, and the use of supplements. In RLS, transcranial stimulation, pneumatic compression, vibrating pads, and acupuncture are emerging interventions. Others, such as aerobic exercise training programs, are known to reduce severity of RLS, enhance QoL, and lower mortality rate in hemodialysis patients. The authors offer a variety of recommendations to be considered in a stepwise management of uremic pruritus, which could be even more effective when taking into account patients' preferences.

In their review, Sars et al. provide well throughout insights into the pathophysiology of intradialytic hypotension (IDH), related outcomes, and their potential impli- 
cations for prevention. Given the complex causality of $\mathrm{IDH}$, it may seem that prevention is unattainable. However, the authors believe that the future on preventing IDH lies in the individualization of dialysis therapy by considering all its clinical aspects in conjunction with smart technology to improve fluid status and maintain tissue perfusion before changes become obviously expressed in IDH.

Han et al. report the results of the HDFIT study, a multicenter randomized controlled trial comparing the effects of hemodiafiltration versus high-flux hemodialysis on self-reported sleep duration. The HDFIT study is the first randomized controlled trial of its kind. Among many other interesting findings, the authors report that patients in the first shift sleep less and experience greater pre-/ posttreatment sleep variability and that operational factors may have greater influence on self-reported sleep duration than patient's demographic or clinical characteristics.

Assessment of fluid status is a basic yet a challenging task. Frank van der Sande et al. provide an interesting description of bioimpedance spectroscopy and its clinical applications. Based on the latest observational studies, the authors conclude that bioimpedance spectroscopy is "an accurate and powerful independent predictor of outcome..." at a population level, asserting that prospective trials in larger hemodialysis and peritoneal dialysis populations are warranted to provide more insights into its clinical utility.

AKI is associated with poor outcomes in critically ill children, particularly in areas where diagnostic and therapeutic resources are limited. Hussein et al. studied the diagnostic utility of the noninvasive saliva urea nitrogen (SUN) dipstick test in Sudanese children suffering from AKI. The authors found a good agreement between the SUN and blood urea nitrogen levels, indicating that SUN could potentially help in timely identifying and monitoring AKI in neonates and children and thus allow for improved treatment.

Access to kidney replacement therapies (KRT) in lowand lower-middle-income countries is limited, and it is estimated that every year over 2 million patients with kidney failure die prematurely because they do not receive KRT due to economic, social, and political factors. Campos et al. are the first to assess the acceptance of allo-hemodialysis (alloHD), a recently proposed technically simple and low-cost KRT (Chao et al. Renal Week ASN 2019, FR-PO0483). In alloHD, the patient's blood flows countercurrent to the blood of a healthy subject ("buddy") through a conventional dialyzer; uremic retention prod- ucts diffuse into the buddy and are excreted by his/her healthy kidneys. In their cross-sectional survey, the authors found that alloHD is accepted by the overwhelming majority of caregivers (87\%) and to a lesser extent by health-care professionals $(60 \%)$. The authors believe that there is an ethical mandate to develop affordable KRT alternatives to improve patients' access and reduce the loss of human lives.

The article by Hanna et al. focuses on defining and assessing long-standing unresolved issues of protein-energy wasting, sarcopenia, and cachexia in CKD patients. Adequate nutrition is particularly crucial to address in hemodialysis and peritoneal dialysis patients where frailty is highly prevalent. The authors propose the malnutrition-inflammation score as a useful tool to predict nutritional risk and provide novel strategies that could be implemented during dialysis in addition to nutritional counseling, for example, dietary intradialytic interventions and the use of exercise tolerance as a marker of frailty.

Rondon-Berrios discusses the spectrum of hyponatremia, which may go unnoticed when it is asymptomatic. Mild hyponatremia can manifest itself through attenuated neurologic deficits, osteoporosis, increased risk of falls, fractures, and increased mortality. Currently, the treatment of syndrome of inappropriate antidiuretic hormone secretion has limited effectivity due to notable therapy side effects, and there are no safe interventions to treat mild hyponatremia. The authors propose increasing plasma sodium levels using urea, which appears to be effective, safe, and well tolerated.

Kazory and Ronco introduce the term "cardiorenal connector" for serum chloride, a frequently ignored electrolyte in patients with heart failure. The authors provide us with the context to interpret current data, along with important implications of correcting serum chloride, making it a potential target of treatment in a subset of patients with heart failure and cardiorenal impairment.

The RRI is dedicated to advancing therapeutic options for CKD patients, providing exceptional care based on research and leading clinical and technological innovations. RRI is fully committed to improve patients' outcomes and QoL. Its active national and international research programs have strong academic alliances with the University of Michigan, Ann Arbor, MI, USA; University of North Carolina, Chapel Hill, NC, USA; University of Rochester, Rochester, NY, USA; Icahn School of Medicine at Mount Sinai, New York, NY, USA; St. Raphael Hospital/Yale, New Haven, CT, USA; Pontifical Catholic 
University of Paraná, Curitiba, Brazil; the University of Guadalajara and the National Heart Institute in Mexico City, Mexico; the University of Maastricht, The Netherlands; the University of Konstanz, Germany; and the University of Graz, Austria, among others.

We hope that you will enjoy the wide range and scope of papers published in this issue of Blood Purification and that you will consider attending next year's 23rd International Conference on Dialysis, Advances in Kidney Disease 2021, which will take place in Las Vegas, Nevada, from January 26 to 29, 2021.

The Editors,

Peter Kotanko, Laura M. Rosales, and Claudio Ronco 